\title{
Evolución reciente y perspectivas de la función de seguridad operacional en el correcto funcionamiento de los aeropuertos
}

\author{
Sara García Ramos \\ Gerente Técnico, Ineco, España \\ Paula Latorre García \\ Técnico, Ineco, España
}

\section{RESUMEN}

La Seguridad Operacional ha ido evolucionando a lo largo de la historia de la aviación especialmente en los últimos años, donde el cambio ha sido más notable dentro los aeropuertos.

La entrada en vigor de normativa que regula la seguridad operacional en los aeropuertos ha supuesto una reinvención del modelo de gestión. Desde formación de personal, pasando por mejora de infraestructuras, hasta la implantación de procedimiento de operación que nos aseguren los niveles de calidad exigidos.

Desde la implantación de la seguridad operacional en los aeropuertos, el mundo de la aviación ha sufrido una de las épocas más revolucionarias, debido a: crisis económicas mundiales, irrupción de nuevos modelos de negocio (compañías "low cost"), operación de nuevos modelos de aeronaves, pérdida de pasajeros a favor de otros medios de trasporte.

Si bien la implantación supuso grandes esfuerzos de concienciación, la evolución no ha sido más fácil ya que obliga al mantenimiento de dichos niveles.

El rápido desarrollo de nuevas tecnologías introduce nuevos factores a tener en cuenta que hasta ahora no había sido necesario contemplar. El avance en los modelos de negocio va enfocado a la construcción de aeronaves cada vez más grandes que deben operar en los aeropuertos ya existentes con todas las garantías de seguridad. El impacto de estos condicionantes implica una dificultad añadida para mantener los estándares de calidad adquiridos, suponiendo un esfuerzo constante y en muchos casos siendo necesario plantear alternativas, por ejemplo estudios aeronáuticos de seguridad que garanticen un nivel de seguridad equivalente.

El propósito del artículo es definir la función de la seguridad operacional en el correcto funcionamiento de los aeropuertos analizando cuales fueron los inicios de la seguridad operacional en el mundo aeronáutico, como se ha implantado, el mantenimiento que requiere y por último adaptación y perspectivas futuras en relación con las nuevas tecnologías.

\section{INICIOS DE LA SEGURIDAD OPERACIONAL}

La seguridad operacional se define como el estado en que el riesgo de lesiones a las personas o daños a los bienes se reduce y se mantiene en un nivel aceptable, o por debajo del mismo, por medio de un proceso continuo de identificación de peligros y gestión de riesgos.

Desde los inicios de la aviación comercial hasta la actualidad, se ha producido un enorme aumento en el número de pasajeros trasportados y operaciones de aeronaves. Este auge en 
el transporte aéreo no hubiera tenido lugar sin el desarrollo de un Sistema de Gestión: seguro, fluido y eficaz.

Sin embargo, el incremento del tráfico aéreo conlleva un aumento en el riesgo de accidentes. Para mantener unos niveles de seguridad adecuados ha sido necesaria una mejora progresiva en la Gestión de la Seguridad Operacional. La Gestión de la Seguridad Operacional no se conforma con mantener la proporcionalidad entre los accidentes y el aumento de tráfico aéreo, ni siquiera con mantener estable la siniestralidad independientemente del incremento de tráfico. Es decir el objetivo es una disminución progresiva del número de accidentes independientemente del crecimiento de tráfico aéreo.

Teniendo en cuenta que:

- La eliminación de todos los accidentes (e incidentes serios) es imposible.

- Los fallos seguirán ocurriendo, a pesar de los más logrados esfuerzos de prevención.

- No hay actividad humana o sistema diseñado por el ser humano que esté totalmente libre de riesgos y errores.

- Los riesgos y errores son aceptables en un sistema implícitamente seguro, siempre que estén bajo control.

Se concluye que la Seguridad Operacional absoluta no existe y además la mitigación de los riesgos requiere de un proceso racional de toma de decisiones. Los sucesos que transcurren tras estas conclusiones llevan a los siguientes acontecimientos:

Creación de la Organización de Aviación Civil Internacional OACI (1944). Objetivo: Fomentar el desarrollo seguro y ordenado de la aviación civil internacional en el mundo.

* Convención de Chicago (1947). Objetivo: Principales normativas internacionales, desarrolladas por (OACI), que recogen requisitos encaminados a alcanzar una adecuada seguridad en el transporte aéreo.

- Anexos (4, 6, 7, 8, 10, 11, 13 y 14).

- Programas GASP (Global Air Safety Programme).

- USOAP (Universal Safety Oversight Aviation Programme).

* Plan Global de Seguridad Aviación (1998). Objetivo: Reducción del No de Accidentes con independencia del crecimiento del número de movimientos.

- Enmienda 40 al Anexo 11 (2001) - Servicios de Tránsito Aéreo.

- PANS-ATM Doc. 4444 Ed. 14 (2001) ("Procedures for Air Navigation Services ICAO Air Traffic Management").

* Desarrollo programas aeropuertos (2001). Objetivo: Implantar el Sistemas de Gestión de Seguridad (SGS).

- Prácticas Estándar y Recomendadas (SARPs-Standard and Recommended Practice ICAO).

- Anexo 14 (5ed 2009/Enm 1-10): Aeródromos.

- Doc 9774 (2001) Manual de Certificación de Aeródromos.

- DOC 9859 (1ed 2005 y 2ed 2009) Manual de Gestión de la Seguridad Operacional. 
El inicio de la seguridad operacional tuvo un denotado carácter técnico, los primeros años OACI centró sus esfuerzos en establecer las normas y reglamentos necesarios para la seguridad operacional de la aviación, su protección y eficacia y la protección del medio ambiente a escala mundial.

Posteriormente la OACI estableció un Plan Global para la Seguridad Aeronáutica (GASP) con el fin de reducir los accidentes e incidentes, no como una actividad independiente sino como un mecanismo que presenta y permite la notificación consolidada de todas esas actividades en un solo documento. En el marco del GASP, la OACI fomenta las numerosas actividades de seguridad operacional que se emprenden en todo el mundo como son facilitar la coordinación de información y experiencia entre los gobiernos y las empresas del sector aeronáutico sobre la seguridad operacional y todos los esfuerzos para asegurar que los programas que se emprenden a escala mundial sean complementarios en lugar de competir entre sí y que aborden las preocupaciones que existen a escala regional y mundial de forma completa, integral y sistemática.

- En el Anexo 11 se señala a la seguridad como el objetivo primario de los servicios de tránsito aéreo (ATS), buscando garantizar una circulación segura, ordenada y expedita del tránsito aéreo; y que se establecerán los niveles y objetivos de seguridad por acuerdos regionales de navegación aérea, cuando corresponda.

- De igual manera, en el Doc. 4444, PANS-ATM, se exponen los objetivos de la gestión de la seguridad para cumplir el nivel de seguridad correspondiente al suministro ATS dentro de un espacio aéreo o en un aeródromo y se expresa que se implantaran mejoras relacionadas con la seguridad siempre que sea necesario. Las disposiciones sobre la implantación de gestión de la seguridad operacional y de programas sistemáticos con niveles y objetivos del Anexo 11 y de los PANS-ATM entraron en vigor desde el 27 de noviembre de 2003.

Por último OACI se centró en el desarrollo de programas para los aeropuertos con el objetivo principal de implantar un Sistema de la Gestión de la Seguridad (SGS). El Sistema de Gestión de Seguridad Operacional es un enfoque sistemático para la gestión de la Seguridad Operacional, que incluye la estructura orgánica, líneas de responsabilidad, políticas y procedimientos necesarios. Como respuesta a esta necesidad, y de acuerdo con las normas y métodos recomendados internacionales relativos a seguridad operacional, OACI elabora el Manual de Gestión de la Seguridad Operacional (SMM) (Doc. 9859), con el fin de proporcionar las disposiciones necesarias para que los Estados desarrollen un programa estatal de seguridad operacional (SPP) bajo el mismo marco.

En la figura 1 se presenta un gráfico en el cual se resume las fases por las que ha trascurrido la seguridad operacional desde el inicio hasta la actualidad: 


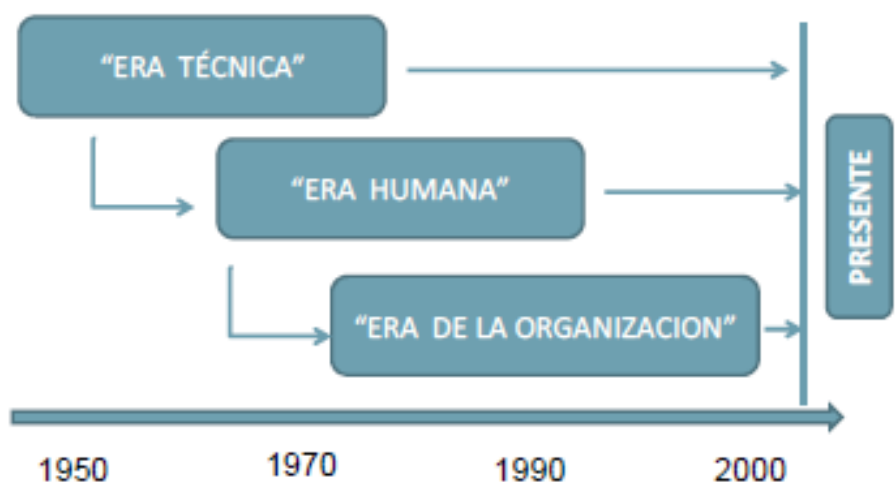

Figura 1 - Evolución de la seguridad operacional.

Los niveles de seguridad operacional de que disfruta hoy el transporte aéreo mundial representan un logro basado en la determinación y esfuerzos de la comunidad aeronáutica en su conjunto.

En el siguiente gráfico se puede observar la disminución progresiva de accidentes por número de movimiento de aeronaves desde la implantación de los primeros Procesos de Gestión de la Seguridad a finales de los 70's.

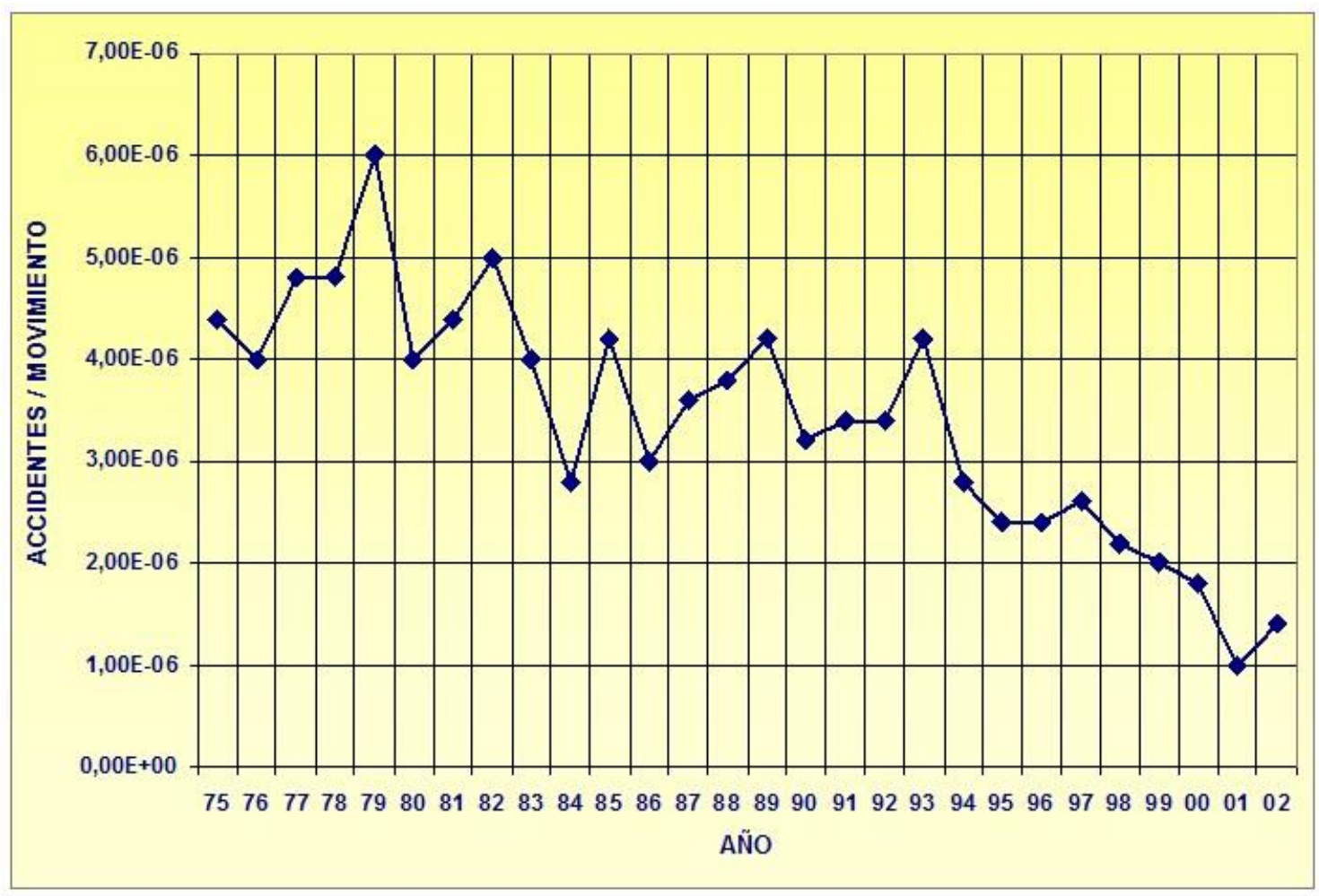

Figura 2 - Evolución del Número de Accidentes Aéreos.

This work is licensed under a Creative Commons Attribution-NonCommercial-NoDerivatives 4.0 International License (CC BY-NCND 4.0). 


\section{Implantación de la Seguridad Operacional}

La Gestión de la Seguridad Operacional ha de ser:

* Sistemática - Las actividades de gestión de la seguridad operacional están de acuerdo a un plan predeterminado y se aplican de manera consistente a través de toda la organización.

* Proactiva - Una aproximación que enfatiza la identificación de los peligros y el control y mitigación de los riesgos, antes que puedan ocurrir eventos que afectan la seguridad.

* Explícita - Todas las actividades de gestión de la seguridad están documentadas y son visibles.

En esta etapa, el trabajo se centra en la implantación de un Sistema de la Gestión de la Seguridad Operacional en los diferentes colectivos aeronáuticos donde es requerido según la normativa que les aplica.

Cabe destacar, que tras haber realizado una primera etapa donde el esfuerzo se basaba en el desarrollo de una normativa internacional, en esta etapa el objetivo prioritario es dar un mayor peso al factor humano en línea con los grandes cambios que debe sufrir las infraestructuras para adaptarse a la nueva normativa.

Actualmente el concepto de Seguridad Operacional es mundialmente conocido y la implantación de un Sistema de Gestión de la Seguridad Operacional es un hecho en los estados miembros de la OACI. Recientemente, los países miembros se encuentran finalizando el proceso de certificación de los aeropuertos y servicios de navegación aérea.

A partir de este punto, quedando aún mucho camino por recorrer, la estrategia a seguir con la implantación es la evaluación constante de los sistemas de gestión de seguridad operacional, manteniendo los objetivos alcanzados hasta el momento y con el propósito de alcanzar los niveles más bajos de riesgo posible, sin olvidar la adaptación progresiva a los cambios que se van produciendo.

\section{Mantenimiento de la Seguridad Operacional}

En el afán de alcanzar un nivel de seguridad óptimo, una vez analizada la responsabilidad en la materialización de incidentes de los factores técnicos y económicos, se hace indispensable fijar el foco de atención en los factores organizativos o institucionales. Este aspecto supone el siguiente punto clave en la consecución y el mantenimiento de los objetivos en materia de seguridad operacional.

Una vez que se han alcanzado los estándares de calidad,

- A nivel técnico: a través de inversión en infraestructuras o implementación de sistemas; $\mathrm{y}$

- A nivel humano: mediante la implantación de procedimientos, reglamentación de la formación y supervisiones del personal,

es imprescindible aunar los esfuerzos en la coordinación a nivel organizativo de toda la estructura aeroportuaria. 
La evolución de la seguridad operacional pasa indispensablemente por entender que todos los implicados en la gestión, explotación y operación de un aeropuerto son colaboradores necesarios que deben participar para lograr un equilibrio entre los factores técnicos y humanos.

El gestor aeroportuario, las compañías aéreas y los proveedores de navegación aérea disponen de sus propios sistemas de gestión de seguridad operacional, pero de poco sirve que cada colectivo persiga sus propios objetivos si no lo hace de manera coordinada con el resto de actores implicados en la operación. Los diferentes sistemas de gestión de la seguridad operacional tienen que integrarse para que formen parte de un engranaje en el que todas las piezas funcionen de manera sincronizada.

Las conclusiones que se obtienen de este planteamiento cambian el enfoque tradicional de la seguridad operacional, orientándola a la normalización de los sistemas de gestión de la seguridad operacional y estableciendo unas directrices comunes.

Para lograr el nuevo planteamiento, es esencial promover la comunicación, de forma que se fomenten espacios de discusión y análisis, donde se ponga en valor la experiencia de todos los participantes. Asimismo, la verdadera coordinación pasa por definir acuerdos con los colectivos internos y externos que determinen las funciones y responsabilidades de cada uno, para que los procedimientos, competencias y el ámbito de actuación queden perfectamente definidos.

Debido a la importancia de la gestión de la seguridad operacional, y surgido de las recomendaciones realizadas durante la celebración de la Conferencia de Directores Generales de Aviación Civil en 2006 y posteriormente en la Conferencia de alto nivel sobre seguridad operacional en 2010, se plantea la importancia de contar con un nuevo Anexo al Convenio sobre Aviación Civil Internacional que recoja en un único documento todos los requisitos aplicables a los Estados en materia de seguridad operacional. Con el fin de reforzar el concepto de que el mantenimiento de las mejoras implantadas hasta el momento sólo son posibles mediante el esfuerzo continuo, la actuación conjunta y la coordinación de todas las partes implicadas. Este proceso culmina en 2013 con la publicación del Anexo 19. Gestión de la Seguridad Operacional.

El resultado de la evolución de todo este proceso lleva a un modelo de explotación aeroportuaria más seguro, del que cabe destacar un alto nivel organizativo logrando de esta manera mayor eficiencia.

\section{Adaptación}

La seguridad operacional debe de ser un proceso dinámico en constante adaptación. La evolución sufrida a través de los distintos cambios de enfoque producidos se debe a la experiencia adquirida a lo largo de los años, pero también a la introducción de avances tecnológicos, que se suman a los factores que se han tenido en cuenta históricamente.

La rápida evolución del transporte aéreo, el aumento del tráfico de pasajeros, la necesidad de establecer rutas de mayor alcance introducen nuevos elementos a los que enfrentarse, como los nuevos modelos de aeronaves de mayor capacidad. No debe olvidarse la búsqueda del mayor beneficio frente al coste manteniendo los mismos niveles de seguridad (objetivo prioritario de las compañías "low cost"). Hacer frente a estos agentes conlleva la revisión y 
actualización continua de los procesos implantados y la introducción de alternativas y mejoras.

La normativa y reglamentación tiene que adaptarse para acoger e implantar las novedades con el objetivo de integrarlas y utilizarlas de manera beneficiosa para el transporte aéreo, logrando que el beneficio económico no esté en detrimento de la seguridad operacional.

Como resultado, se crean nuevas recomendaciones internacionales que buscan mantener el nivel de seguridad a través de la implantación de nuevos procedimientos. Este es el caso del reciente documento de OACI, Procedimientos para los servicios de navegación aérea Aeródromos (PANS-Aeródromos) (Doc. 9981), primera edición 2015. Este documento detalla los procedimientos operacionales que deben aplicar los explotadores de aeródromos para garantizar la seguridad operacional en especial cuando no es posible cumplir plenamente las especificaciones técnicas requeridas. Citando textualmente el alcance y aplicación del documento:

"El contenido de los PANS-Aeródromos está diseñado para permitir que los procedimientos y metodologías descritos en el documento se empleen para evaluar los problemas operacionales que enfrentan los aeródromos existentes en un entorno cambiante y que plantea desafíos y resolver esos problemas, a fin de garantizar la seguridad permanente de las operaciones de los aeródromos".

Como se ha visto, atribuir a los factores técnicos y humano todas las posibilidades de mejora de un sistema en materia de seguridad operacional, significa no haber comprendido que no existe un sistema perfecto que esté libre de peligros. Por eso, la evolución de la seguridad operacional busca relacionar los costes con la mejora de la seguridad operacional, con el objetivo de encontrar un equilibrio entre ambos que genere el menor impacto socioeconómico posible sin comprometer el nivel de seguridad equivalente.

\section{Futuro}

Al mismo tiempo que la innovación en el mundo de la aviación supone nuevos retos de cara a mantener el nivel de seguridad operacional, también puede aportar mejoras, dando solución a los problemas de infraestructuras existentes en los aeropuertos, proporcionando sistemas de medición cada vez más precisos o resolviendo las deficiencias que no era posible adecuar debido a problemas físicos, o a falta de recursos técnicos o económicos.

Por ejemplo, en la actualidad no está permitido que los drones sobrevuelen espacio aéreo controlado. Si en un futuro se permitiera su introducción, supondría un gran desafío de cara a la integración con el resto de operaciones y al mantenimiento de la seguridad operacional, sin embargo, podría aportar grandes ventajas en campo de las inspecciones, vuelos de calibración de ayudas visuales, control de obstáculos u operaciones de emergencia, búsqueda y salvamento. 


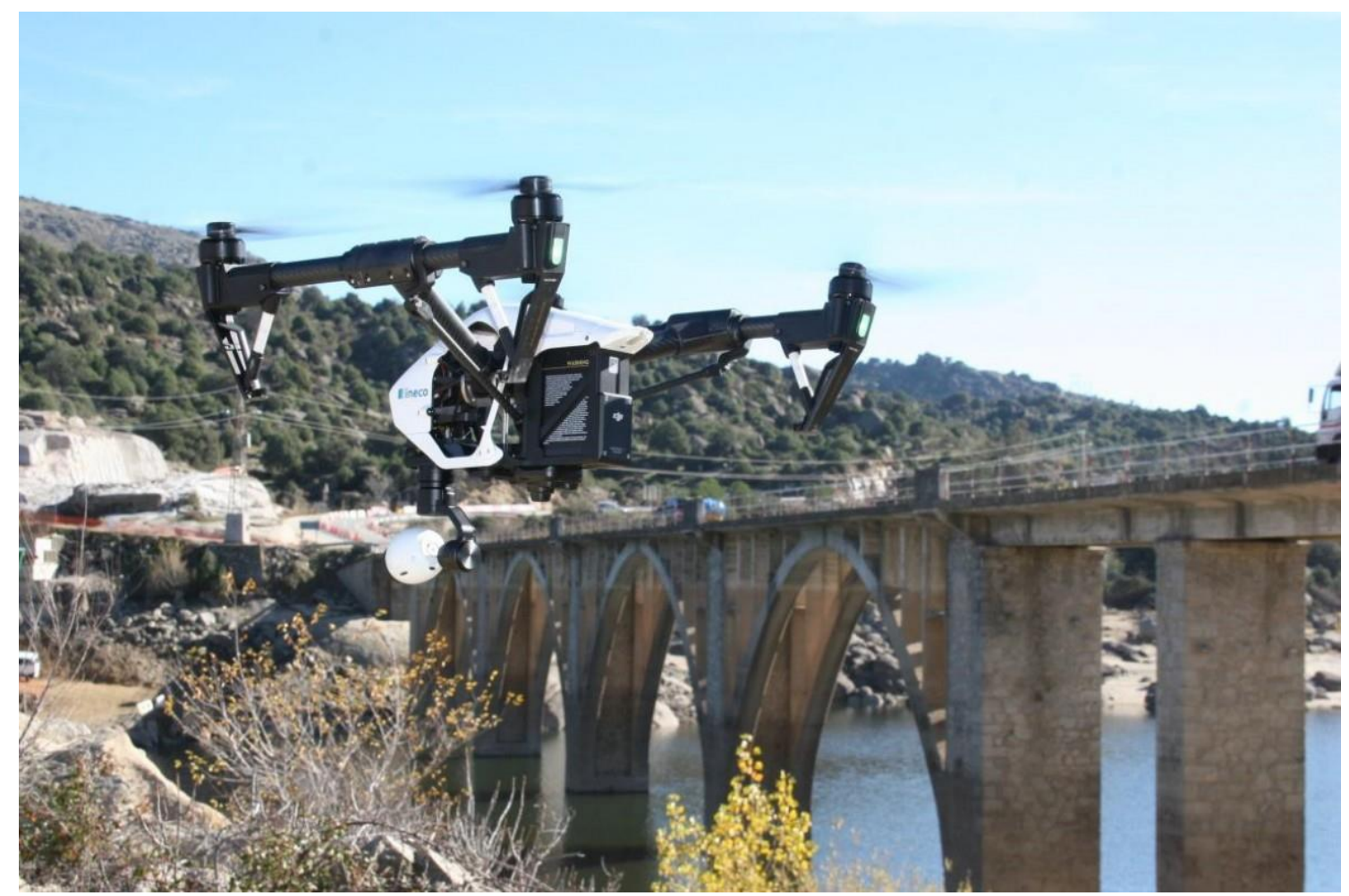

Figura 3 - Dron capaz de realizar fotografía, filmaciones y levantamientos aéreos (levantamientos topográficos, fotogrametría).

Otros ejemplos destacables, que han implicado cambios en la seguridad operacional son:

- Procedimientos de coordinación frente al TCAS (Traffic alert and Collision Avoidance System)

- Los Sistema de frenado de emergencia (EMAS Engineered materials arresting system)

- Procedimientos frente a cenizas volcánicas

\section{CONCLUSIONES}

Las ideas que se destacan en el artículo son:

- La seguridad operacional es un proceso continuo en el tiempo que se va alimentando con los cambios que se van produciendo.

- El coste (económico, operacional, medio ambiental, etc.) de cualquier actuación frente al beneficio en la seguridad operacional debe estar equilibrado de manera que no se produzca un coste desproporcionado en comparación con el beneficio obtenido en la seguridad operacional. 